\title{
Asiaticoside alleviates cardiomyocyte apoptosis and oxidative stress in myocardial ischemia/reperfusion injury via activating the PI3K-AKT-GSK3 $\beta$ pathway in vivo and in vitro
}

\author{
Xueliang Zeng ${ }^{1}$, Junjian Yu ${ }^{2}$, Peipei Liu ${ }^{1}$, Yuan Liu ${ }^{1}$, Taohui Zeng ${ }^{1}$, Bei Li ${ }^{1}$ \\ ${ }^{1}$ Department of Pharmacy, First Affiliated Hospital of Gannan Medical University, Ganzhou, China; ${ }^{2}$ Cardiovascular and Thoracic Surgery \\ Department 2, First Affiliated Hospital of Gannan Medical University, Ganzhou, China \\ Contributions: (I) Conception and design: B Li; (II) Administrative support: X Zeng; (III) Provision of study materials: J Yu; (IV) Collection and \\ assembly of data: P Liu; (V) Data analysis and interpretation: T Zeng; (VI) Manuscript writing: All authors; (VII) Final approval of manuscript: All \\ authors. \\ Correspondence to: Bei Li. Department of Pharmacy, First Affiliated Hospital of Gannan Medical University, 23 Qingnian Road, Ganzhou 341000, \\ China. Email: gyfylibei2009@163.com.
}

Background: Myocardial ischemia/reperfusion (MI/R) is one of the most important links in myocardial injury, causing damage to cardiac tissues including cell apoptosis, oxidative stress, and other serious consequences. Asiaticoside (AS), a new compound synthesized from genistein, is cardioprotective. This paper presents new evidence for the protective role of AS against MI/R injury in vitro and in vivo.

Methods: First, BALB/c mice underwent surgical ligation of the left anterior descending (LAD) artery to establish an MI/R animal model, and HL-1 cells were subjected to oxygen-glucose deprivation/ reperfusion (OGD/R) to establish an in vitro model. Myocardial infarct size was examined by triphenyl tetrazolium chloride (TTC) staining, histopathological changes detected in heart tissues were observed using hematoxylin and eosin (H\&E) and Masson staining, heart tissue apoptosis was assessed by terminal deoxynucleotidyl transferase dUTP nick end labeling (TUNEL) staining. Enzyme-linked immunosorbent assay (ELISA) kits were used to analyze cardiac troponin I $(C T n I)$, creatine kinase-muscle and brain $(C K$ $M B)$, lactate dehydrogenase $(L D H)$, superoxide dismutase $(S O D)$, malondialdehyde $(M D A)$, and reduced glutathione $(G S H)$. Cell viability was evaluated using Cell Counting Kit-8 (CCK-8) and live/dead assay. Cell apoptosis, reactive oxygen species (ROS), mitochondrial membrane potential, and mitochondrial superoxide were detected by flow cytometry and fluorescence microscopy. Both the protein expression in myocardial tissues and cardiomyocytes were examined by western blot.

Results: In the in vivo MI/R experiments, pretreatment of AS reduced myocardial infarct size, decrease leakage of myocardial enzyme, suppressed myocardial apoptosis, myocardial collagen deposition, and oxidative stress. In the in vitro OGD/R experiments, HL-1 cells pretreated with AS had increased cell viability, decreased apoptosis rates and depolarization of mitochondrial membrane potential, and attenuated intracellular ROS and mitochondrial superoxide. Moreover, AS downregulated the expression of apoptotic protein, and promoted phosphorylation of $P I 3 K, A K T$, and GSK3 $\beta$, which was reversed by $P I 3 K$ inhibitor LY294002.

Conclusions: The AS compound protects against MI/R injury by attenuating oxidative stress and apoptosis via activating the $P I 3 K / A K T / G S K 3 \beta$ pathway in vivo and vitro.

Keywords: Asiaticoside (AS); ischemia/reperfusion injury; oxidative stress; apoptosis; PI3K pathway

Submitted Nov 23, 2021. Accepted for publication Jan 11, 2022.

doi: 10.21037/atm-21-6667

View this article at: https://dx.doi.org/10.21037/atm-21-6667 


\section{Introduction}

Ischemic heart disease (IHD) is a common clinical cardiovascular disease that threatens the health of many populations worldwide (1). Although IHD can be treated by drugs or surgery, reperfusion causes more severe myocardial injury, which is termed myocardial ischemia/reperfusion (MI/R) injury (2). Several adverse cardiovascular outcomes result from MI/R injury, such as coronary artery spasm, cardiac dysfunction, and cardiac arrest (3-5). Hence, it is necessary to search for novel therapeutic strategies and drugs to improve clinical outcomes of patients with MI/R.

Asiaticoside (AS) is a natural triterpenoid compound mainly extracted from Centella asiatica, and has a wide range of pharmacological actions (6). Centella asiatica has been widely used in the clinical application in India. Further, the leaves of Centella asiatica has been used in tea in Indian daily life. AS has a sweet aroma. Oral taken AS 1 $\mathrm{g} / \mathrm{kg}$ is safe for adult (7). AS could induce type-I collagen synthesis to promote the repair of skin (8). Extensive pharmacological research have demonstrated that AS exerts anti-inflammatory (9), anti-tumor (10), anti-oxidant (11), and anti-fibrosis (12) effects, among others. The latest research showed the protective effect of AS against cerebral I/R injury via downregulating the NOD2/MAPK/ $N F-\kappa B$ pathway in rats (13). Previously confirmed the cell protection role of AS on oxygen-glucose deprivation (OGD) injured H9c2 cardiomyocytes (14). However, whether AS has a protective effect during MI/R-induced cardiac injuries remains need to be explored in vivo and in vitro.

Phosphatidylinsitol-3-kinase $(P I 3 K)$-protein kinase $\mathrm{B}$ $(A K T)$ is a signal transduction pathway regulating various cellular functions such as cell proliferation, survival, metabolism, and DNA double-strand break (DSB) repair (15-17). Members of the PI3K family include 2 subunits regulatory subunit, regulatory subunit $\mathrm{p} 85 \alpha$, and catalytic subunit $\mathrm{p} 110 \alpha$, that are mediators of the classic $P I 3 K / A K T$ pathway signaling (18). The $P I 3 K$ members do not recruit and activate $A K T$ directly, but through generation of second messenger lipid phosphatidylinositol (3-5)-triphosphate (PIP3) to serve this function. However, the occurrence of these posttranslational modifications of $A K T$ is strictly dependent on $P I 3 K$ activity (19). Glycogen synthase kinase $3(G S K 3)$ is a multifunctional serine/threonine kinase found in all eukaryotes, which consists of 2 main isoforms: GSK3 $\alpha$ and $G S K 3 \beta$. Furthermore, GSK3 $\beta$ is a downstream signaling molecule of the PI3K/AKT pathway (20). Activation of $A K T$ increases the phosphorylation of $G S K 3$ and leads to inactivation of $G S K 3 \beta$, which plays a crucial role in regulating cell growth and apoptosis (21). Previously, it has been shown that activation of the $P I 3 K / A K T /$ $G S K 3 \beta$ pathway has a beneficial effect on various ischemic conditions, such as myocardial ischemia (22), cerebral ischemia (23), and hepatic ischemia (24).

In the present study, we established an in vivo model of $\mathrm{MI} / \mathrm{R}$ and in vitro models of oxygen-glucose deprivation/ reperfusion $(\mathrm{OGD} / \mathrm{R})$, with the aim of investigating the myocardial protective effects of AS on I/R injury, and exploring the crucial role of the $P I 3 K / A K T / G S K 3 \beta$ pathway in this process. We present the following article in accordance with the ARRIVE reporting checklist (available at https:// atm.amegroups.com/article/view/10.21037/atm-21-6667/rc).

\section{Methods}

\section{Experimental animals and drug administration}

A total of $24 \mathrm{BALB} / \mathrm{c}$ mice males 8-10 weeks old, weight 20-22 g, purchased from Shanghai SLAC Laboratory Animal Co., Ltd. [License number: SCXK (Shanghai, China) 2017-0005]\}. Experiments were performed under a project license (No. LLSC-2020090801) granted by the Gannan Medical University Institutional Animal Care and Use Committee, in compliance with the Guide for the Humane Treatment of Laboratory Animals (Ministry of Science and Technology of the People's Republic of China, Policy No. 2006398) for the care and use of animals. For each treatment, mice were randomized as follows: mice of low-dose $(n=6)$ and high-dose $(n=6)$ groups were intraperitoneally injected with 1 and $2 \mathrm{mg} / \mathrm{kg}$ AS per day, respectively, for 7 consecutive days before MI/ R. The mice of the MI/R model group $(n=6)$ and sham group $(n=6)$ received an intraperitoneal injection of equal volume of physiological saline. One mg AS (HY-N0439, MedChemExpress, USA) was dissolved in $1 \mathrm{~mL} 10 \%$ $\mathrm{DMSO}+90 \%$ corn oil mixture to prepare storage liquid. The storage liquid was dilute with $10 \%$ DMSO + 90\% corn oil mixture to form work reagent.

\section{Preparation of MI/R model}

The MI/R surgery was performed as previously described (25). Briefly, mice were anesthetized with $2 \%$ isoflurane during procedures. Subsequently, left thoracotomy and pericardotomy were conducted to expose the heart. A slipknot was tied around the left anterior descending (LAD) coronary artery $3-4 \mathrm{~mm}$ from its origin utilizing a 6-0 silk suture to induce myocardial ischemia. 
After $30 \mathrm{~min}$ of ischemia, the slipknot was released to reperfuse the myocardium for $12 \mathrm{~h}$. In the sham group, mice were subjected to the same surgical procedures without ligating the LAD coronary artery.

\section{Measurement of myocardial infarction area}

Triphenyl tetrazolium chloride (TTC) staining was used to assess infarct size. The hearts were quickly removed to a $-80{ }^{\circ} \mathrm{C}$ refrigerator for $20 \mathrm{~min}$. Then, the frozen hearts were sectioned transversely into $2 \mathrm{~mm}$ thick slices and incubated in $1 \%$ TTC (Sigma Aldrich, St. Louis, MO, USA) at $37^{\circ} \mathrm{C}$ for $20 \mathrm{~min}$. The stained tissue sections were fixed in $4 \%$ paraformaldehyde and pictures were taken with a Nikon digital camera (Nikon, Tokyo, Japan). Pictures were analyzed using Image J software (https://imagej.nih.gov/ij/).

\section{Observation of myocardial pathomorphology}

Histopathology was conducted by hematoxylin and eosin $(\mathrm{H} \& \mathrm{E})$ staining and Masson staining. Briefly, the cardiac tissues were fixed in $10 \%$ formaldehyde, dehydrated by gradient ethanol, embedded in paraffin, and sectioned at $4 \mu \mathrm{m}$ thickness. Then, tissue sections were stained with H\&E Staining Kit (Beyotime, Shanghai, China) and Masson staining kit (Solarbio, Beijing, China). The operations were conducted strictly according to the instructions and evaluated using a BX43 light microscope (Olympus, Tokyo, Japan).

\section{Terminal deoxynucleotidyl transferase dUTP nick end labeling (TUNEL) staining}

We used a TUNEL staining kit (Roche, Basel, Switzerland) to detect apoptosis in cardiac tissue. Briefly, the prepared slices of tissues were incubated with $100 \mu \mathrm{L}$ of proteinase $\mathrm{K}$ for $10 \mathrm{~min}$ and washed with phosphate-buffered saline (PBS). After the glass slide was air-dried, the slices of tissues were incubated in a solution of terminal deoxynucleotidyl transferase (TdT) with dUTP mixture at $37^{\circ} \mathrm{C}$ for $1 \mathrm{~h}$. After 3 washes with PBS, $100 \mu \mathrm{L}$ diaminobenzidine (DAB) substrates were added for reaction at $20{ }^{\circ} \mathrm{C}$ for $10 \mathrm{~min}$. Finally, the slides were observed by light microscopy.

\section{Detecting myocardial enzymes}

Biochemical indicators of myocardial injury and oxidative stress were used to diagnose the MI/R, including cardiac troponin I $(C T n I)$, creatine kinase-muscle and brain $(C K$ $M B)$, lactate dehydrogenase $(L D H)$ content in serum, superoxide dismutase $(S O D)$, malondialdehyde $(M D A)$, and reduced glutathione $(G S H)$ content in tissue. The above assay kits were all purchased from Jiancheng Bioengineering Institute (Nanjing, China). These indices were measured according to the manufacturer's instructions respectively and the results were read on a microplate absorbance reader (Molecular Devices, San Jose, CA, USA).

\section{Preparation of OGD/R model and drug treatment}

Mouse HL-1 cardiomyocytes were purchased from Sigma Aldrich and provided with high glucose Dulbeccos' modified Eagle medium (DMEM; Gibco, New York, NY, USA) containing $10 \%$ fetal bovine serum (FBS; Gibco), $100 \mathrm{U} / \mathrm{mL}$ streptomycin (Beyotime), and $100 \mathrm{U} / \mathrm{mL}$ penicillin (Beyotime) in a humidified incubator at $37{ }^{\circ} \mathrm{C}$, $5 \% \mathrm{CO}_{2}$. To establish the OGD/R model, the cells were cultured in glucose/serum-free DMEM (Solarbio) and placed in hypoxic incubator $\left(5 \% \mathrm{CO}_{2}, 95 \% \mathrm{~N}_{2}\right)$ for $12 \mathrm{~h}$ to induce OGD. After that, the medium was replaced with normal culture medium and the plates were incubated in a normoxic chamber for $12 \mathrm{~h}$ of reoxygenation. The low and high concentrations of AS (50 and $200 \mu \mathrm{g} / \mathrm{mL}$ ) and 10 mol/L LY294002 (MCE, Monmouth, NJ, USA) were used alone or in combination for $12 \mathrm{~h}$ before OGD/R stimulation. Normal HL-1 cardiomyocytes were cultured in DMEM without any treatment and served as a negative control.

\section{Cell survival analysis}

Cell survival was assessed performed using the Cell Counting Kit-8 assay (CCK-8; Dojindo, Shanghai, China). The HL-1 cells were seeded at 5,000 cells/well into 96-well plates. After different treatments, the culture medium was discarded and $0.1 \mathrm{~mL}$ of CCK- 8 working solution was added to each well. After $1.5 \mathrm{~h}$ of incubation, absorbance was measured at $450 \mathrm{~nm}$ on a microplate absorbance reader. The cell survival rate was expressed as a percentage of the control.

\section{Live/dead cell staining}

After relevant treatments, HL-1 cells were rinsed twice using PBS and covered with $0.1 \mathrm{~mL}$ serum-free medium per well. Then, cells were stained with $2 \mu \mathrm{M}$ calcein-AM 
(Dojindo, Shanghai, China) and $5 \mu \mathrm{M}$ propidium iodide (PI; Dojindo) and incubated at $37^{\circ} \mathrm{C}$ for 30 min protected from light. Representative images were captured on the IX73 fluorescence microscope (Olympus).

\section{Assessment of cell apoptosis}

An annexin V-FITC/PI apoptosis kit (Multi Sciences, Hangzhou, China) was used to detect apoptosis by flow cytometry. Briefly, cells from each group were digested with $0.25 \%$ ethylenediamine tetraacetic acid (EDTA)-free trypsin and washed twice with PBS. The cells were counted and the cell density was adjusted to $1 \times 10^{6}$ cells $/ \mathrm{mL}$. Immediately after, $5 \mu \mathrm{L}$ of Annexin V-FITC and $10 \mu \mathrm{L}$ PI were added and incubated for $5 \mathrm{~min}$ at $4{ }^{\circ} \mathrm{C}$ in the dark in preparation for apoptosis analysis on an Accuri C6 flow cytometer [Becton, Dickinson and Co., (BD) Biosciences, San Jose, CA, USA].

\section{Assessment of the mitochondrial membrane potential $(\Delta \Psi m)$}

A mitochondrial membrane potential kit (Beyotime) was utilized to test mitochondrial membrane potential. According to the manufacturer's instructions, $2 \times 10^{5} \mathrm{HL}-1$ cells were stained with JC-1 for 20 min protected from light. After washing in PBS, the fluorescence intensity (red/ green) was detected using flow cytometry.

\section{Determination of cell reactive oxygen species (ROS)}

The level of intracellular ROS was assessed using an ROS assay kit (Beyotime). Briefly, HL-1 cells were washed with PBS and incubated in serum-free medium containing $10 \mu \mathrm{mol} / \mathrm{L}$ of dichloro-dihydro-fluorescein diacetate (DCFH-DA) at $37{ }^{\circ} \mathrm{C}$ for $20 \mathrm{~min}$. Then, cold PBS was used to wash away the extracellular DCFH-DA molecules. The DCFH-DA fluorescence was monitored using flow cytometry and observed under a fluorescence microscope.

\section{Determination of mitochondrial superoxide}

MitoSOX Red Superoxide Indicator (Invitrogen, Carlsbad, CA, USA) was used to detect ROS levels. The HL-1 cells were incubated in PBS with $2 \mu \mathrm{mol} / \mathrm{L}$ MitoSox Red reagent for $30 \mathrm{~min}$ at $37^{\circ} \mathrm{C}$ in dark. Cells were then washed with
PBS and analyzed on a flow cytometer and observed under a fluorescence microscope.

\section{Protein extraction and western blot assay}

Briefly, cytosolic and mitochondrial proteins were separately extracted from myocardial tissues and HL-1 cells using Mitochondrial/Cytoplasmic Protein Extraction Kit (Beyotime) according to the manufacturer's instructions. The lysates were boiled for 10 min after a cocktail of protease inhibitors (Beyotime) had been added. After separation on a precast Express ${ }^{\mathrm{TM}}$ PAGE gels (GeneScript, Nanjing, China) by electrophoresis, denatured protein samples were transferred onto a polyvinylidene fluoride (PVDF) membrane (Millipore, Billerica, MA, USA). The membranes were then blocked using $5 \%$ bovine serum albumin (BSA; Beyotime) for $1 \mathrm{~h}$ and incubated with the following antibodies at $4{ }^{\circ} \mathrm{C}$ overnight: $B c l-2$ (ab196495, 1:1,000), Bax (ab32503, 1:5,000) (all from Abcam, Cambridge, MA, USA), cleaved caspase-3 (9664, 1:1,000), cleaved caspase-9 (9507, 1:1,000), Cyto-c (11940, 1:1,000), PI3K (13666, 1:1,000), p-PI3K (17366, 1:1,000), AKT (4685, 1:2,000), p-AKT (4060, 1:2,000), GSK3 $\beta$ (12456, 1:1,000), p-GSK3 $\beta(5558,1: 1,000)$ (all from Cell Signaling Technology, Boston, MA, USA), GAPDH (AF5009, 1:5,000, Beyotime) and COX IV (4850, 1:1,000, CST) were used as a loading control. After washing with tris-buffered saline with Tween 20 (TBST) thrice (10 min each), the membrane was probed with horseradish peroxidase (HRP)-conjugated antirabbit IgG (A0208, Beyotime) or anti-mouse IgG (A0216, Beyotime) secondary antibodies at room temperature for $1 \mathrm{~h}$ and washed thrice with TBST. The signal was then developed using electrochemiluminescence (ECL; Beyotime) and imaged on a chemiluminescence detection system (Bio-Rad Laboratories, Hercules, CA, USA). Band intensities were analyzed by Image J.

\section{Statistical analysis}

All data were shown as mean \pm standard deviation (SD) and were analyzed with GraphPad Prism 6.0 (GraphPad Inc., La Jolla, CA, USA). Comparisons between groups were assessed using Student's $t$-test. One-way analysis of variance (ANOVA) was used to compare differences among more than three groups, and Tukey test was used for post hoc analysis. A $\mathrm{P}$ value $<0.05$ indicated statistical significance. 

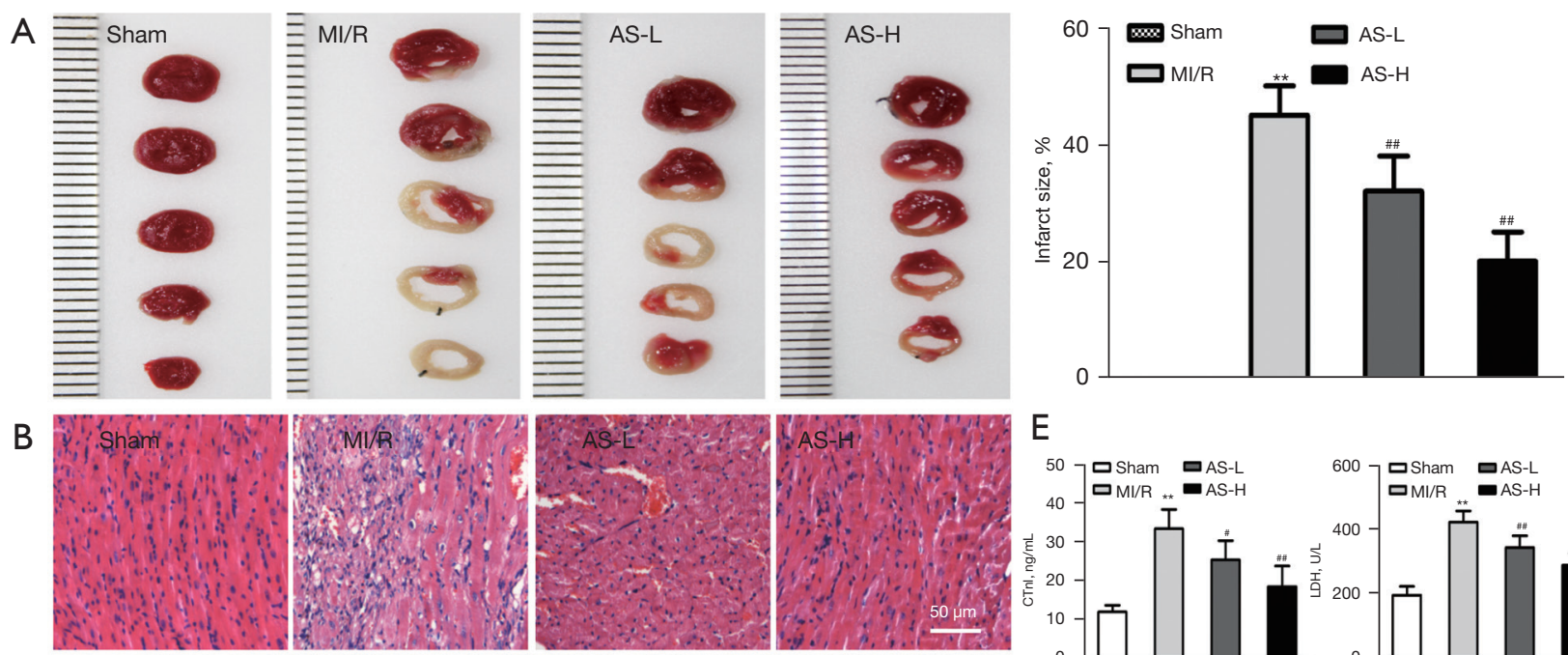

E
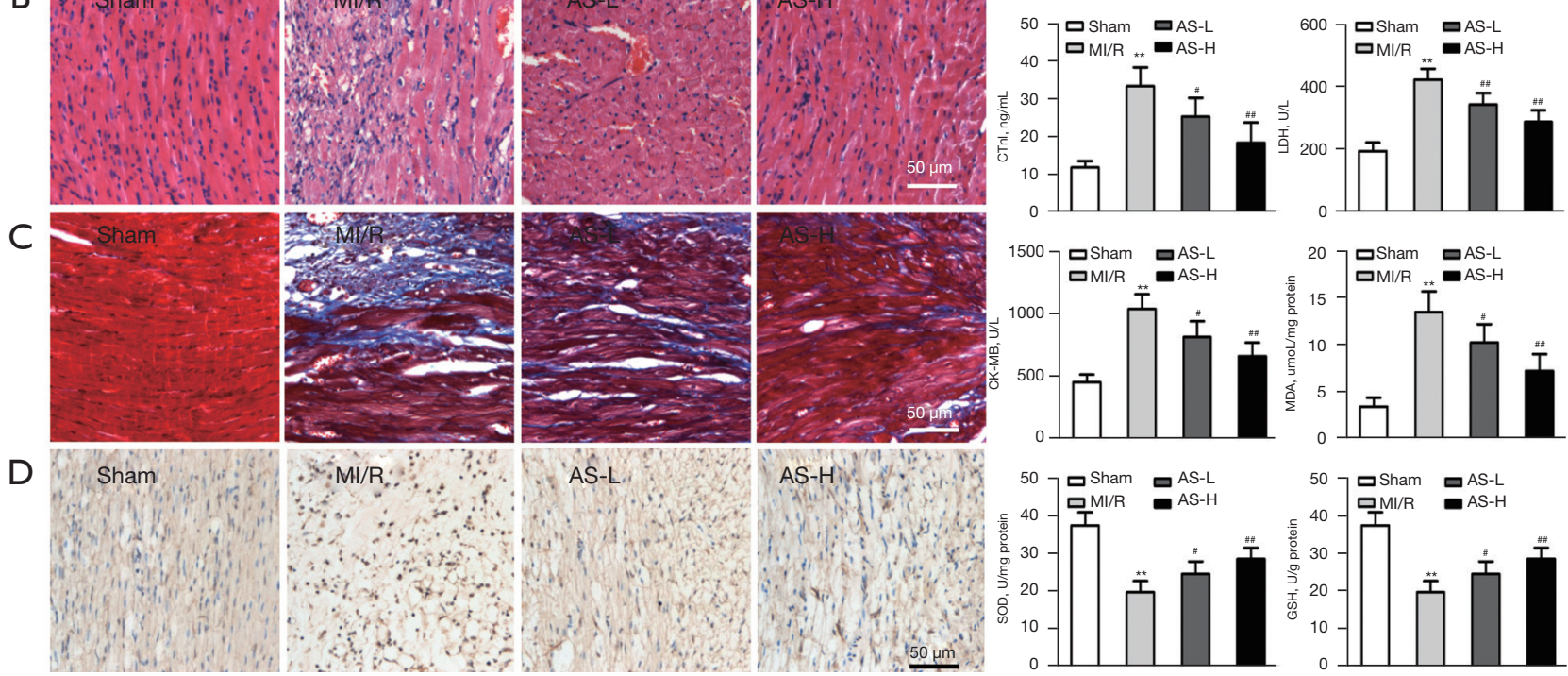

Figure 1 Different doses of AS preconditioning protect MI/R injury in mice. (A) Representative TTC staining of mouse heart sections and quantification analysis of the infarct size. (B) Histopathological observation of H\&E staining on heart sections of mice. (C) Collagen deposition of heart assessed by Masson staining, fibrotic area is stained blue. (D) Representative pictures of TUNEL staining (positive cardiomyocyte nuclei stained in brown). (E) Concentration of CTnI, CK-MB, LDH in serum, and SOD, MDA, and GSH in heart tissue. Error bars indicate means $\pm \mathrm{SD}$ of 3 independent experiments. ${ }^{* *} \mathrm{P}<0.01, \mathrm{MI} / \mathrm{R}$ group $v s$. sham group; ${ }^{*} \mathrm{P}<0.05$ or ${ }^{* \#} \mathrm{P}<0.01$, various dosages of AS pretreatment groups vs. MI/R group. AS, asiaticoside; MI/R, myocardial ischemia/reperfusion; TTC, triphenyl tetrazolium chloride; $\mathrm{H} \& \mathrm{E}$, hematoxylin and eosin; TUNEL, terminal deoxynucleotidyl transferase dUTP nick end labeling; CTnI, cardiac troponin I; $C K-M B$, creatine kinase-muscle and brain; $L D H$, lactate dehydrogenase; $S O D$, superoxide dismutase; $M D A$, malondialdehyde; $G S H$, glutathione; SD, standard deviation; L, low-dose; H, high-dose.

\section{Results}

\section{AS protected against MI/R in mice}

Before the experiment, there was no significant difference in animal behavior and state between the groups. According to the analysis of data from TTC staining, AS significantly decreased infract size in AS pretreatment groups than MI/R group (Figure 1A). Results of histopathological examination following $\mathrm{H} \& \mathrm{E}$ and Masson staining showed that inflammatory cell infiltration and the fibrotic area were significantly increased in the MI/R group compared to the sham group; in contrast, inflammatory cell infiltration and fibrotic alterations in heart were dramatically improved when MI/R mice were pretreated with AS (Figure 1B,1C). The TUNEL assay showed that apoptosis of cardiac myocytes was significantly increased following MI/R, while AS pretreatment evoked a marked decrease in the number of apoptotic cells (Figure 1D). To further evaluate the extent of myocardial injury, biochemical indicators of myocardial injury were also studied. Serum levels of CTnI, $L D H$, and $C K-M B$ were strongly increased in the MI/R group compared with the sham group, and AS significantly 
inhibited $C T n I, L D H$, and $C K-M B$ caused by MI/R (Figure $1 E$ ). In order to confirm the antioxidative effects of AS, we assessed levels of $M D A, S O D$, and GSH. Compared with the sham group, there was a marked elevation of $M D A$, and decreased activity of $S O D$ and $G S H$ in the MI/R group; AS significantly inhibited the $M D A$ content and enhanced $S O D$ and GSH levels in serum of MI/R mice (Figure $1 E$ ). All these data demonstrate that AS alleviated MI/R-induced cardiomyocyte apoptosis and oxidative stress in mice.

\section{AS exerted the cardioprotective effect by activating the PI3K/AKT/GSK3ß pathway in MI/R mice}

The apoptotic process is complex and regulated by a wide range of proteins (26). To assess the role of AS in MI/ R-injury, we assayed the expression of several apoptosisrelated proteins. Our results revealed that compared with the sham group, Bax, cleaved caspase-3, cleaved caspase-9, and Cyto-c (cytosolic) protein expression levels significantly increased, while Bcl-2 and Cyto-c (mitochondria) significantly decreased in the MI/R group. However, AS pretreatment partially reversed the changes in protein expression induced by MI/R injury (Figure $2 A$ ). The $P I 3 K / A K T / G S K 3 \beta$ pathway is an important pathway for cell survival and involved in the regulation of cell apoptosis (27). We examined the phosphorylation of the $P I 3 K / A K T / G S K 3 \beta$ pathway in mice with $\mathrm{MI} / \mathrm{R}$ by western blot (Figure 2B). Compared with the sham group, the levels of phosphorylated PI $3 K, A K T$, and GSK $3 \beta$ were slightly increased in the I/R group, this may have been due to the stress reaction as a result of $M I / R$. The pretreatment further significantly upregulated the phosphorylation of $P I 3 K, A K T$, and GSK3 $\beta$ in the MI/R group. We also observed that the expression levels of the total PI3K, AKT, and GSK $\beta$ showed no significant changes all groups. These results may suggest that AS promotes activation of the $P I 3 K / A K T / G S K 3 \beta$ signal pathway in MI/R-injury.

\section{AS attenuated cytotoxicity induced by OGD/R and reversed OGD/R-induced apoptosis in HL-1 cardiomyocytes}

To investigate the relative contribution of AS in OGD/ $\mathrm{R}$ injury in vitro, we tested cell survival after OGD/R in the presence or absence of different concentrations of AS pretreatment. The CCK-8 analysis revealed that cell survival was significantly inhibited following OGD/R. In contrast, pretreatment with AS improved the survival rate of OGD/R-exposed HL-1 cells (Figure $3 A$ ). The CCK-8 result was also confirmed by live/dead staining (Figure 3B). Next, we detected cell apoptosis by flow cytometry. Flow cytometric analysis confirmed that OGD/ $\mathrm{R}$ obviously increased the rate of apoptosis whereas pretreatment with AS reduced cell apoptosis (Figure 3C). Additionally, the JC-1 result showed that AS pretreatment markedly decreased the OGD/R-induced formation of JC-1 monomers, which indicated that AS restored the OGD-induced loss of mitochondrial membrane potential (Figure 3D).

\section{AS ameliorated OGD/R-triggered oxidative stress}

To determine the role of AS on cellular OGD/R-induced oxidative stress in vitro, the levels of intracellular ROS in HL-1 cells were determined by flow cytometry and fluorescence microscope. The ROS levels were appreciably enhanced in the OGD/R group compared with the control group; however, the AS pretreated group displayed significantly decreased ROS levels compared with the OGD/R group (Figure 3E,3F). Next, we also evaluated the mitochondrial-specific ROS levels in vitro using MitoSOX red, a mitochondrial superoxide indicator. Flow cytometric data showed that AS pretreatment appreciably ameliorated OGD/R-induced mitochondrial ROS production in HL-1 cells, compared with the OGD/R group (Figure $3 G$ ). This result was further confirmed by corresponding immunofluorescence pictures (Figure $3 H$ ).

\section{AS protected $O G D / R$-induced injury by triggered the activation of the PI3K/AKT/GSK3 $\beta$ pathway}

Firstly, we measured the change in proteins related to apoptosis pathway. Western blot results showed that Bax, cleaved caspase-3, cleaved caspase-9, and Cyto-c (cytosolic) protein expression levels significantly increased, $B c l-2$ and Cyto-c (mitochondria) significantly decreased in the OGD/R group compared to the control group. When compared with the OGD/R group, pretreatment with AS partially reversed these changes (Figure $4 A$ ). Moreover, the phosphorylation of PI3K, AKT, and GSK3 $\beta$ in the AS pretreatment group was further acutely elevated than in the OGD/R group; meanwhile, no significant changes of $A K T$ protein were observed in the groups (Figure $4 B$ ). Consistent with the results in vitro, these findings suggested that the ameliorative effects of AS on OGD/R-induced injury were 

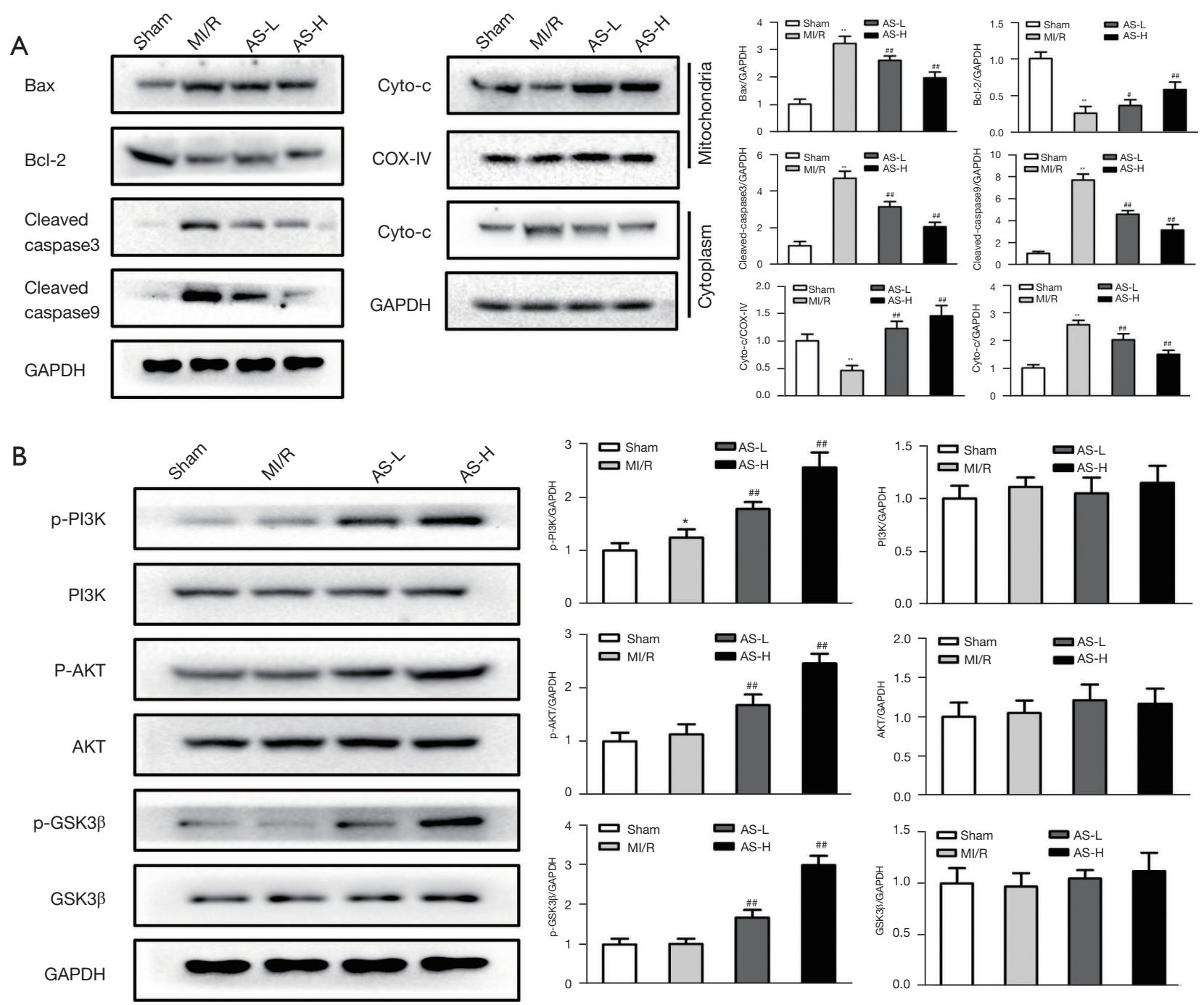

Figure 2 Effect of AS on protein expression in cardiac tissue after MI/R in mice. (A) The expression levels of apoptosis-related proteins were measured by western blot in different heart tissues. (B) The protein and phosphorylation levels of PI3K, AKT, and GSK3 $\beta$ were detected by western blot in different heart tissues. The band intensities were quantified using Image J software and normalized to GAPDH or COX-IV. Error bars indicate means $\pm \mathrm{SD}$ of three independent experiments. ${ }^{*} \mathrm{P}<0.05,{ }^{* *} \mathrm{P}<0.01$, MI/R group vs. sham group; ${ }^{\#} \mathrm{P}<0.05$ or ${ }^{\#} \mathrm{P}<0.01$, various dosages of AS pretreatment groups vs. MI/R group. AS, asiaticoside; MI/R, myocardial ischemia/reperfusion; SD, standard deviation; L, low-dose; H, high-dose.

associated with the $P I 3 K / A K T / G S K 3 \beta$ pathway.

\section{LY294002 inbibited the protection effect of $A S$}

To further confirm whether the PI3K/AKT/GSK3 $\beta$ pathway participates in protective effect of AS, LY294002 $(10 \mu \mathrm{mol} / \mathrm{L})$ was combined with AS in OGD/R HL-1 cells. We found that the cell viability was even lower than AS group (Figure 5A); meanwhile, cell death, apoptosis and formation of JC-1 monomers were significantly increased compared to AS group (Figure 5B-5D). These results suggest that LY294002 may inhibit the protective effect of AS on OGD/R.

\section{LY294002 inbibited antioxidative stress effects of AS}

Flow cytometric analysis showed that LY294002 combined with AS significantly increased the levels of intracellular 

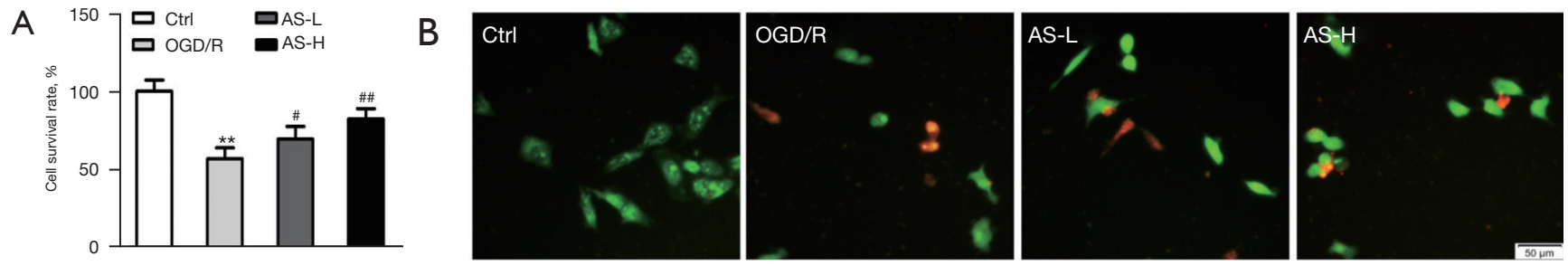

\section{Ctrl}

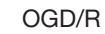

AS-L

AS-H

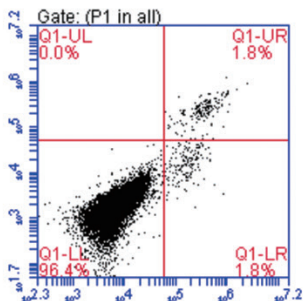

F Gate: (P1 in all)
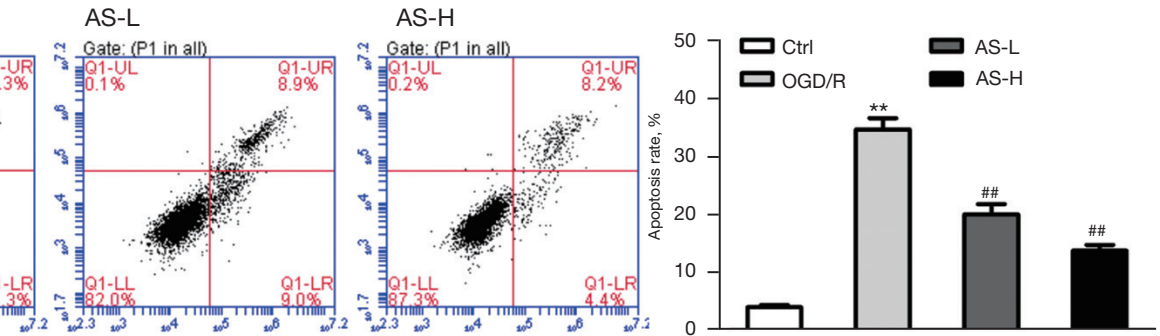

D Ctrl

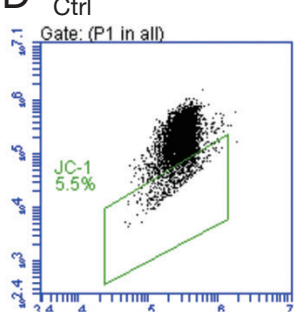

$\mathrm{OGD} / \mathrm{R}$

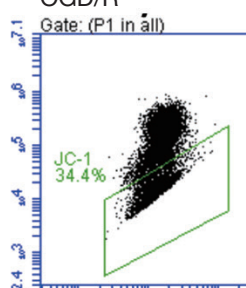

AS-L

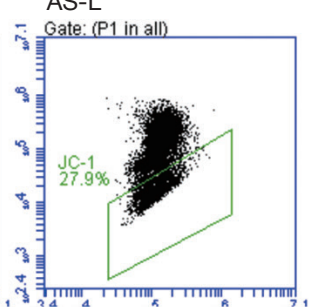

AS-H
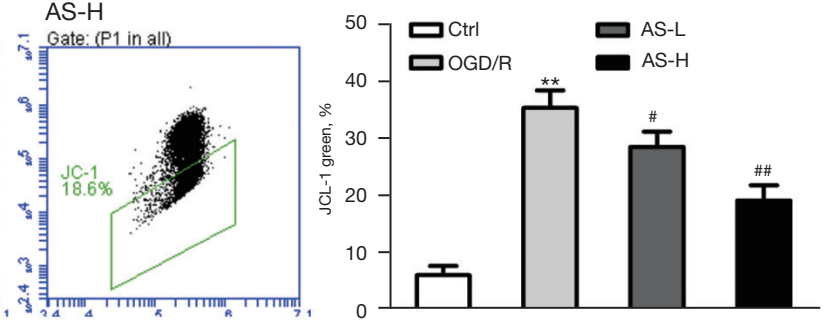

\section{E Ctrl}

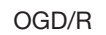

o Gate: ( $P 1$ in all)

으 Gate: (P1 in alli)

$$
\text { AS-L }
$$
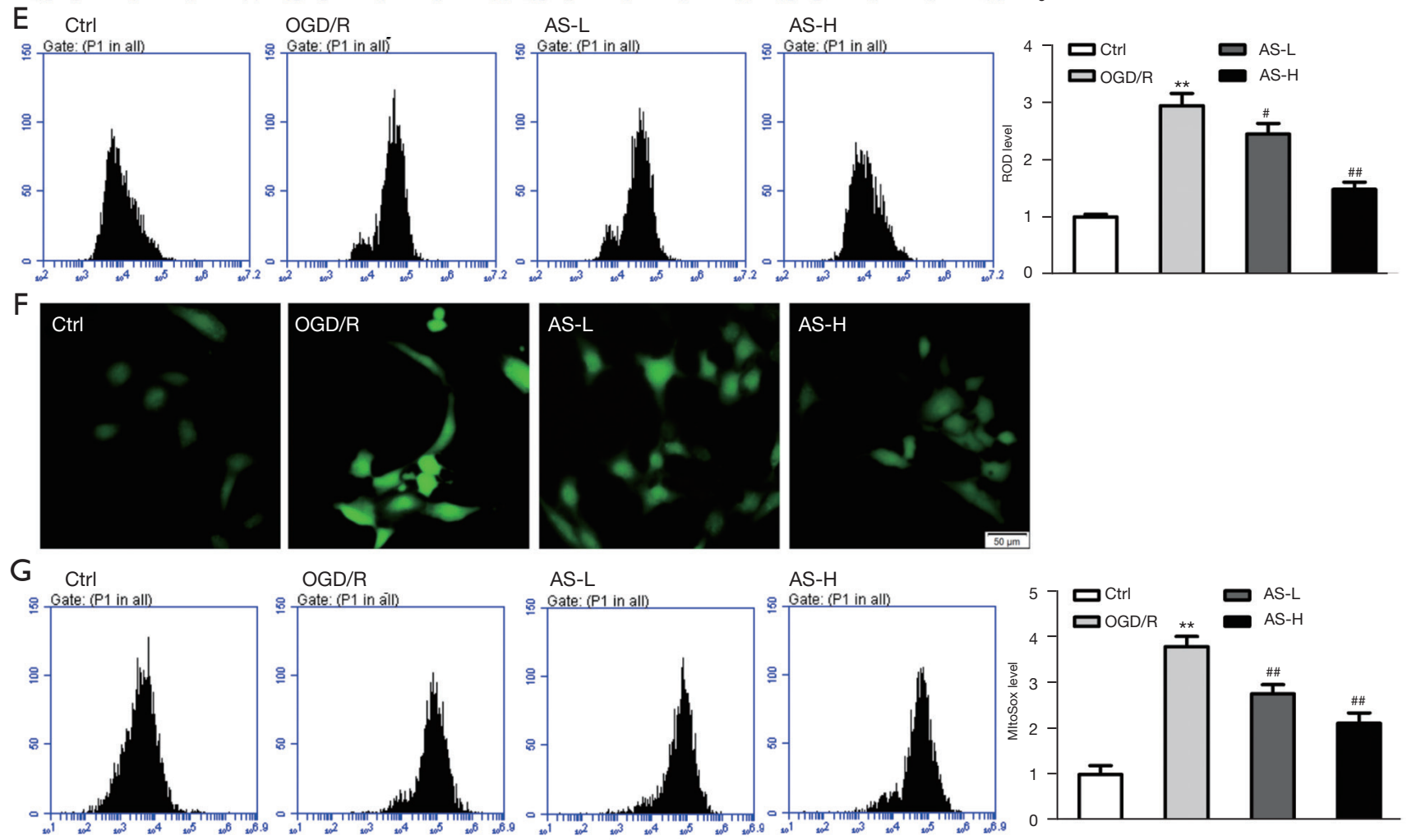

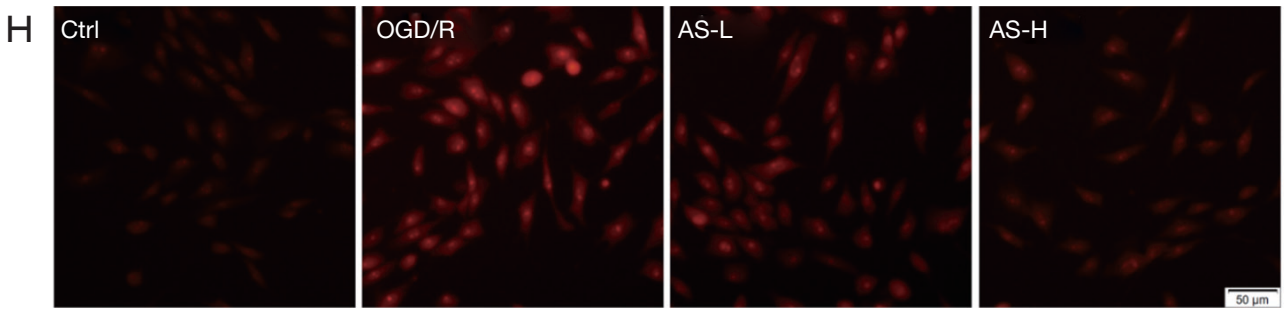

Figure 3 AS protects against OGD/R-induced injury in HL-1 cardiomyocytes. (A) Cell survival rates were detected by the CCK-8 assay. (B) Fluorescence microscopy images of live/dead staining (green staining indicates viable cells, red staining indicates dead cells). (C) Cell apoptosis was evaluated using flow cytometry. (D) Depolarization of mitochondrial membrane potential was analyzed by flow cytometry. (E,F) The level of intracellular ROS was assessed using flow cytometry, representative images of ROS staining were captured under a fluorescent microscope. $(\mathrm{G}, \mathrm{H})$ The level of mitochondrial superoxide was determined using flow cytometry, representative images of MitoSOX staining were captured under a fluorescent microscope. Error bars indicate means $\pm \mathrm{SD}$ of 3 independent experiments. ${ }^{* *} \mathrm{P}<0.01, \mathrm{OGD} / \mathrm{R}$ group $v s$. control group; ${ }^{\#} \mathrm{P}<0.05$ or ${ }^{\# \#} \mathrm{P}<0.01$, various dosages of AS pretreatment groups $v$ s. OGD/R group. AS, asiaticoside; OGD/R, oxygen-glucose deprivation/reperfusion; CCK-8, Cell Counting Kit-8; ROS, reactive oxygen species; SD, standard deviation; L, low-dose; H, high-dose.

ROS compared with the single treatment of AS (Figure 5E, $5 F$ ). Additionally, the decreases in mitochondrial ROS levels in AS group were abrogated by treatment with LY294002 in combination (Figure 5G,5H). The above results suggested that antioxidative stress effects of AS were partly blocked by LY294002.

\section{LY294002 reversed the effect of AS on the PI3K/AKT/ GSK3ß pathway}

To assess the relevance of the $P I 3 K / A K T / G S K 3 \beta$ pathway activation in the protective effect of AS, we performed western blot analysis. The results showed that $B a x$, cleaved caspase-3, cleaved caspase-9, and Cyto-c (cytosolic) protein expression levels significantly increased, and $B c l-2$ and Cyto-c (mitochondria) significantly decreased in the AS + LY294002 group compared to the AS group (Figure 6A). This demonstrates that the combination pretre atment of AS and LY294002 led to weaker anti-apoptotic effects of AS. The western blot analysis also revealed a marked reduction of $\mathrm{p}-P I 3 K, \mathrm{p}-A K T$, and $\mathrm{p}-G S K 3 \beta$ expression in the AS + LY294002 group relative to the AS group (Figure 6B). Together, these data demonstrated that LY294002 suppressed the benefits of AS on OGD/R damage by blocking the $P I 3 K / A K T / G S K 3 \beta$ pathway.

\section{Discussion}

Myocardial ischemic injury has become one of the major diseases that endangers human health and life, and there is still a lack of safe and effective drugs and treatments.
The AS compound is a natural triterpenoid compound mainly extracted from Centella asiatica which demonstrates pharmacological effects including anti-apoptosis and antioxidant, but the role of AS in MI/R injury treatment is undefined (28). In this study, we utilized in vivo and in vitro models to investigate the protective effect of AS against MI/ $\mathrm{R}$-induced cardiac damage. We found that AS pretreatment conferred cardioprotective effects, as evidenced by improved MI/R cardiac functional recovery, reduced myocardial apoptosis, and attenuated oxidative stress. More importantly, the $P I 3 K / A K T / G S K 3 \beta$ pathway was shown to play a significant role in this process. This study provided new evidence that AS could be useful in the development of a novel strategy against IHD.

The markers of $C T n I, C K-M B$, and $L D H$ are sensitive myocardial injury measurements used for the diagnosis and detection of myocardial injury (29). These diagnostic serum marker enzymes of cardiotoxicity liberate out of the cardiac tissue into blood circulation due to myocardial damage (30). Currently, TTC staining, a commonly used to quantify experimental tissue infarctions, is the gold standard for measuring lesion sizes in pre-clinical studies of myocardial infarction (31). In our experiments, the clear visible myocardial infarct size and significant increase in CTnI, CK$M B$, and $L D H$ suggested the successful establishment of an MI/R model. Correspondingly, after pretreatment with AS, these phenomena decreased in varying degrees, indicating that AS has a significant cardioprotective effects.

In normal physiological states, collagen deposition and inflammatory response of heart tissue is devastatingly low, but it is significantly expanded and contributes to 

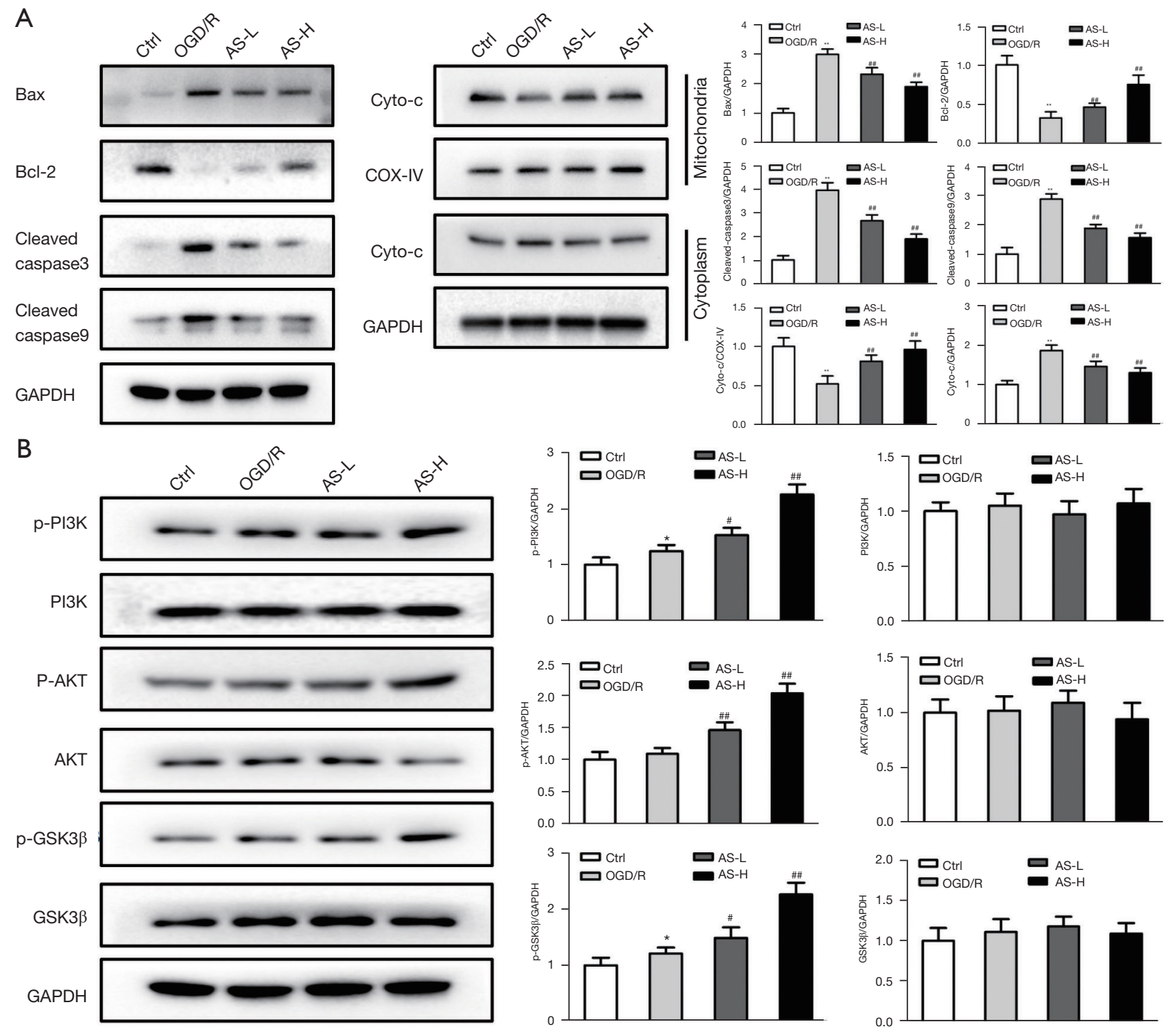

Figure 4 Effect of AS on protein expression in HL-1 cardiomyocytes after OGD/R-induced injury. (A) The expression levels of apoptosisrelated proteins were measured by western blot in HL-1 cells with various treatments. (B) The protein and phosphorylation levels of $P I 3 K, A K T$, and GSK3 $\beta$ were detected by western blot in differently treated HL-1 cells. The band intensities were quantified using Image $\mathrm{J}$ software and normalized to GAPDH or COX-IV. Error bars indicate means $\pm \mathrm{SD}$ of 3 independent experiments. ${ }^{*} \mathrm{P}<0.05$, ${ }^{*} \mathrm{P}<0.01$, OGD/R group vs. ctrl group; ${ }^{\#} \mathrm{P}<0.05$ or ${ }^{\# \#} \mathrm{P}<0.01$, various dosages of AS pretreatment groups vs. OGD/R group. AS, asiaticoside; OGD/R, oxygen-glucose deprivation/reperfusion; SD, standard deviation; L, low-dose; H, high-dose.

further damage in disease states, such as MI, cardiac hypertrophy, and heart failure $(32,33)$. Thus, evaluation of histopathological changes in the cardiac tissue using Masson and $H \& E$ staining is necessary. The cardiac pathology results showed that AS markedly attenuated inflammatory cell infiltration and the accumulation of collagen deposition in MI/R mice, which further validated the protective effects to the infarcted heart induced by AS.
Cardiomyocytes are terminally differentiated cells, which rarely undergo apoptosis in normal physiology (34). Apoptosis, a highly programmed process of cell death, is regulated by numerous molecular signaling pathways (35). Increasing evidence has indicated that apoptosis caused by I/R results in myocyte injury and that inhibiting cardiomyocyte apoptosis leads to cardioprotection (36). In fact, inhibition of myocardial 

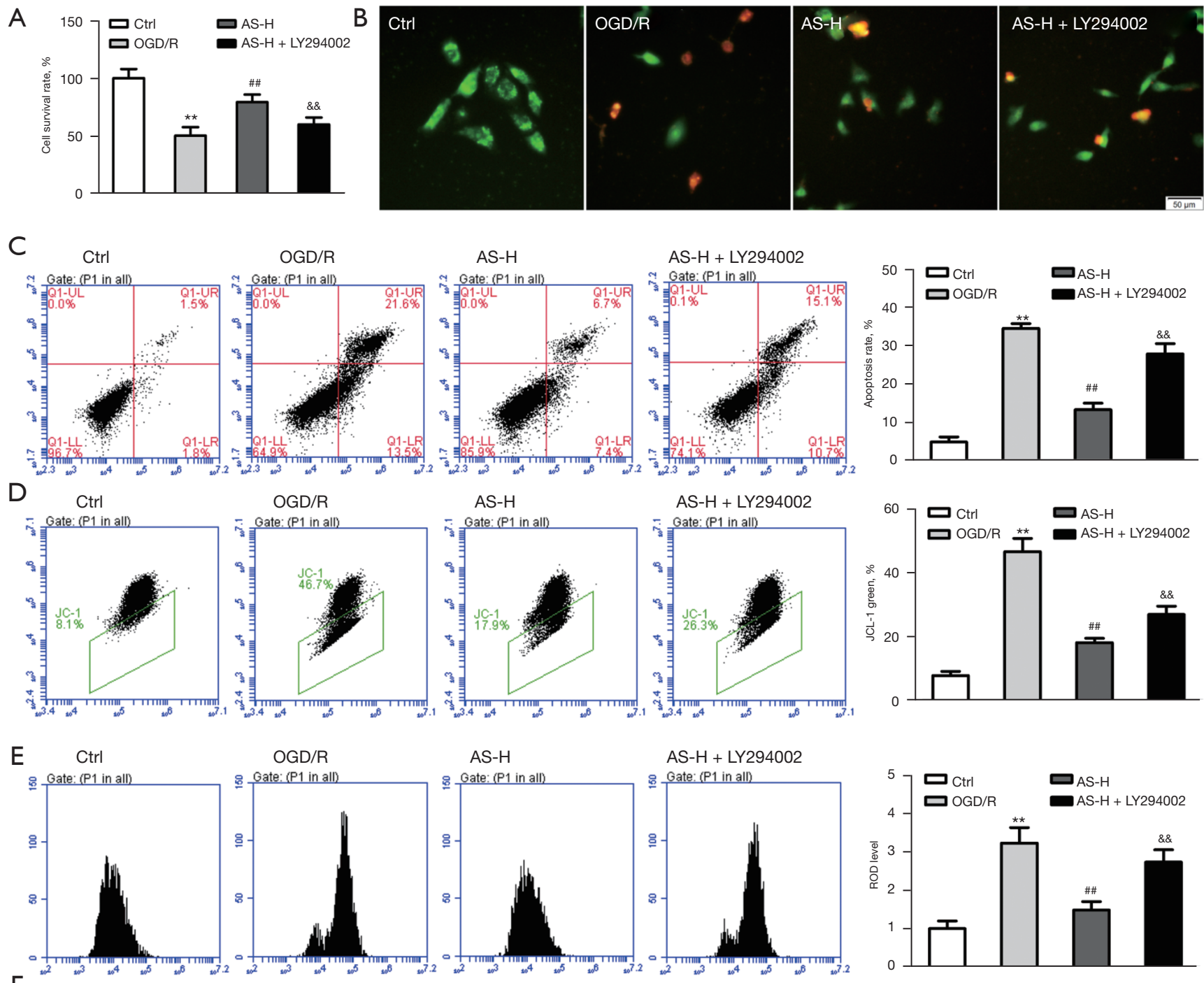

AS-H

AS-H + LY294002

$\mathrm{F}$
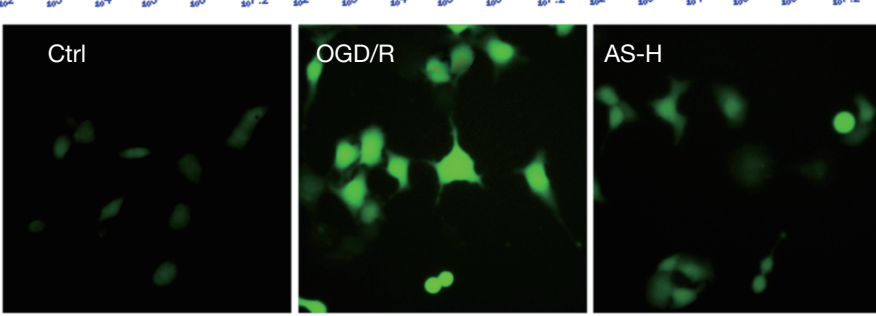

G
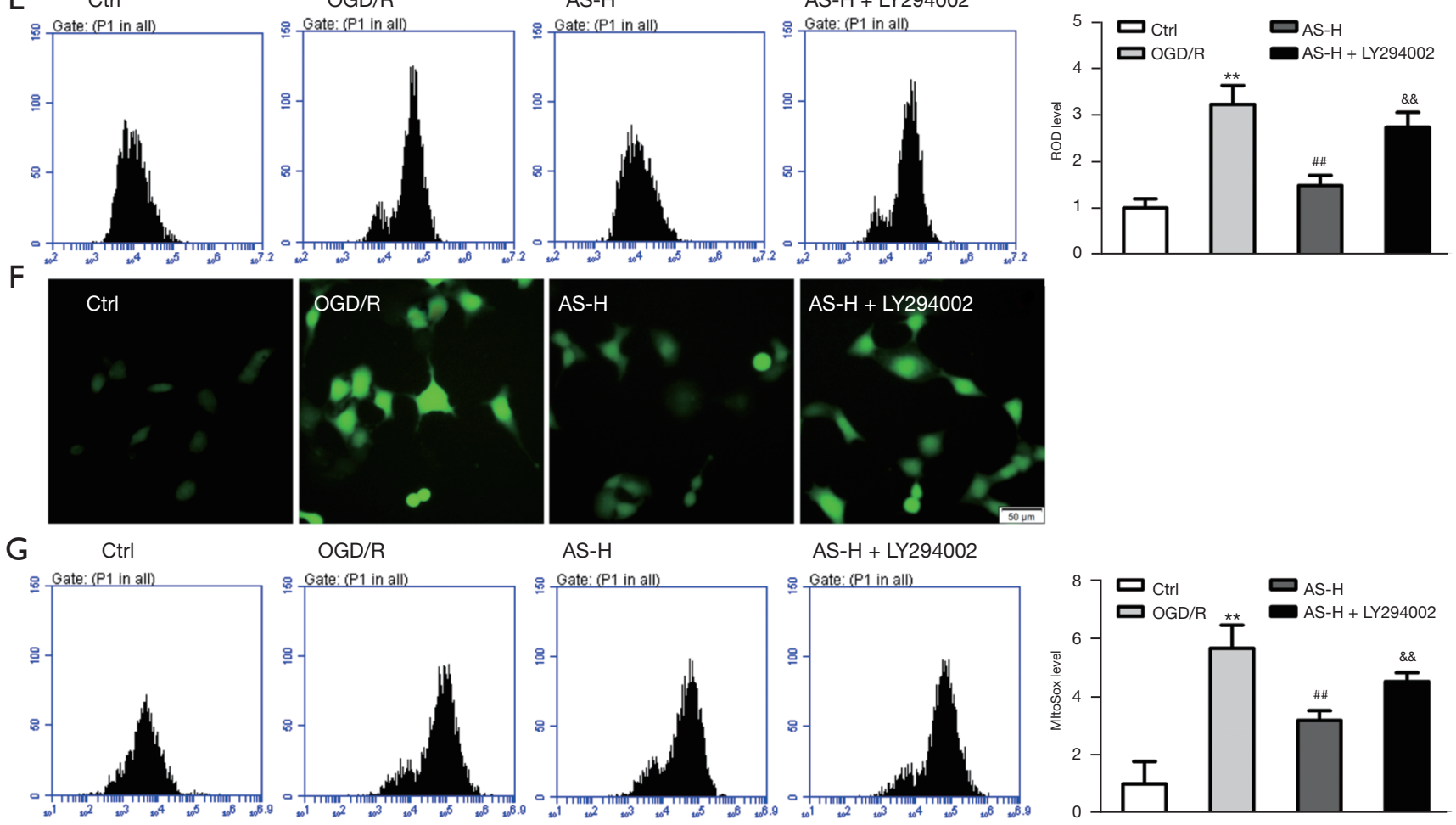

AS-H

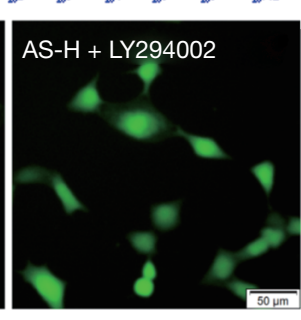

AS-H + LY294002

Gate: (P1 in all)
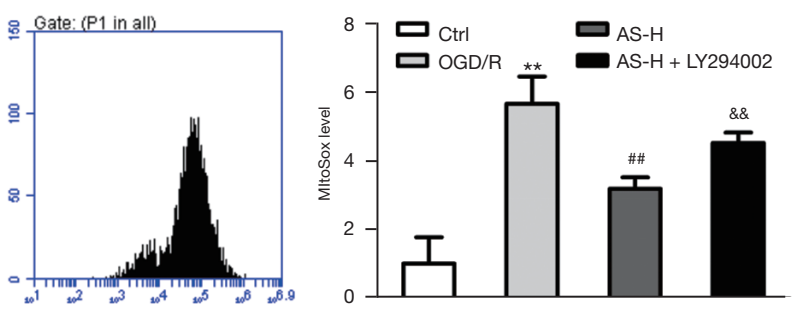

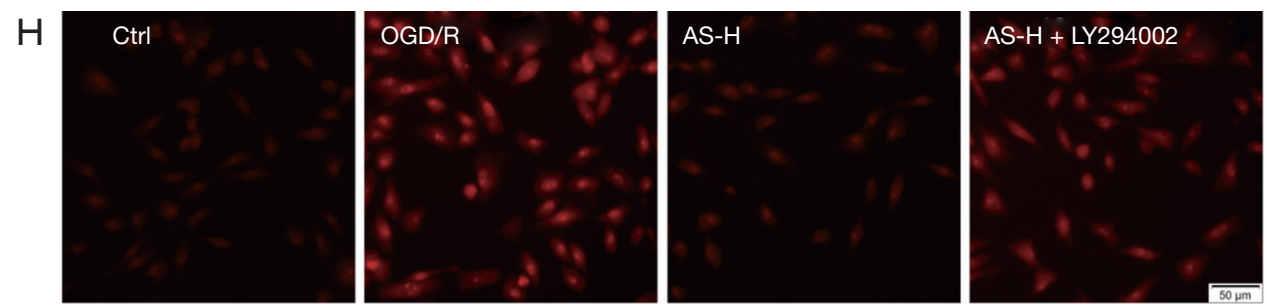

Figure 5 LY294002 partially reverses the protective effects exerted by AS against OGD/R-induced destruction in HL-1 cardiomyocytes. (A) Cell survival rates were detected by the CCK-8 assay. (B) Fluorescence microscopy images of live/dead staining (green staining indicates viable cells, red staining indicates dead cells). (C) Cell apoptosis was evaluated using flow cytometry. (D) Depolarization of mitochondrial membrane potential was analyzed by flow cytometry. (E,F) The level of intracellular ROS was assessed using flow cytometry, representative images of ROS staining were captured under a fluorescent microscope. $(\mathrm{G}, \mathrm{H})$ The level of mitochondrial superoxide was determined using flow cytometry, representative images of MitoSOX staining were captured under a fluorescent microscope. Error bars indicate means \pm $\mathrm{SD}$ of 3 independent experiments. ${ }^{* *} \mathrm{P}<0.01$, OGD/R group vs. control group; ${ }^{\# \#} \mathrm{P}<0.01$, AS-H group vs. OGD/R group; ${ }^{\& \&} \mathrm{P}<0.01, \mathrm{AS}-\mathrm{H}$ + LY294002 group vs. AS-H group. AS, asiaticoside; OGD/R, oxygen-glucose deprivation/reperfusion; ROS, reactive oxygen species; SD, standard deviation; L, low-dose; H, high-dose.

apoptosis is a common mechanism of many natural products. It has been reported that Picroside II ameliorates myocardial morphology and decreased infarct size by inhibiting cardiomyocyte apoptosis (37). Ginsenoside Rg1 could protect diabetic rats from myocardial injury through attenuation of myocardial apoptosis, possibly by inhibiting the expression of caspase 3 and restoring $B c l-x L$ (38). Futhermore, puerarin- $V$ restored the function of myocardial cells by downregulating apoptosis in cardiac tissue (39). In this research, we found that pretreatment with AS could reduce the apoptosis of cardiomyocytes in the MI/R mice by TUNEL staining. In parallel, AS inhibited apoptosis rate in HL-1 cells after OGD/R. It may be that the change of apoptosis-related and anti-apoptosis-related proteins in I/R injury can be partially overcome by AS, which has been demonstrated by western blot both in vivo and vitro.

Oxidative stress is closely associated with inflammation, apoptosis, and energy metabolism as well as the basic pathophysiological process responsible for the development of (40). The enzyme activities of $M D A, S O D$, and $G S H$ are related to oxidative stress, among which $M D A$ is an indicator of lipid peroxidation and $S O D$ and $G S H-P x$ are endogenous antioxidant enzymes (41). In order to investigate the balance between oxidation and antioxidation in MI/R mice, we measured the levels of $M D A, S O D$, and $G S H$. The present results showed that MI/R led to an imbalance in the antioxidant defense system, which reduced the activities of SOD and $G S H$ and improved the $M D A$ level in the heart. This effect was reversed by the pretreatment of AS. In addition, oxidative stress results from an imbalance between capacity to remove ROS and ROS accumulation in cells, and it is necessary to target intracellular ROS and mitochondrial ROS to prevent cell apoptosis caused by oxidative stress (42). In the present study, we used 2 types of fluorescent regents to detect oxidative stress status in HL-1 cells exposed to OGD/R, one was DCFH-DA, for intracellular total ROS and the other was MitoSOX Red, especially for mitochondrial superoxide. Then, we found inhibition of overproduction of ROS and mitochondrial superoxide with AS pretreatment attenuated obviously OGD/R-induced oxidative stress in HL-1 cells. Taken together, AS has been shown to exert a protective effect against oxidative stress both in vivo and vitro.

Some signaling molecules have been shown to be cytoprotective, which promote the survival of the cells from insultation, including $P I 3 K, A K T$, and $G S K 3 \beta$ (43-45). The $A K T$ molecule is the responsory of $P I 3 K$ and its activation strongly enhances cell resistance ability to apoptotic stimulus (46). As the key downstream gene of $A K T, G S K 3 \beta$ could regulate many important cell processes, including cell proliferation, apoptosis, and energy metabolism. The phosphorylated $G S K 3 \beta$ is the inactive type. $G S K 3 \beta$ activation inhibition is the critical process in myocardial adaptation $(47,48)$. In this study, we found that AS treatment greatly promoted the expression of $\mathrm{p}-P I 3 K, \mathrm{p}-A K T$, and $\mathrm{p}-G S K 3 \beta$ in a dose-dependent manner both in vivo and vitro. The beneficial role of AS was evidently restricted by LY294002, an PI3K inhibitor. These results confirmed the role of the $P I 3 K / A K T$ pathway in AS treating MI/R.

In conclusion, this study clarified the treatment effect of 

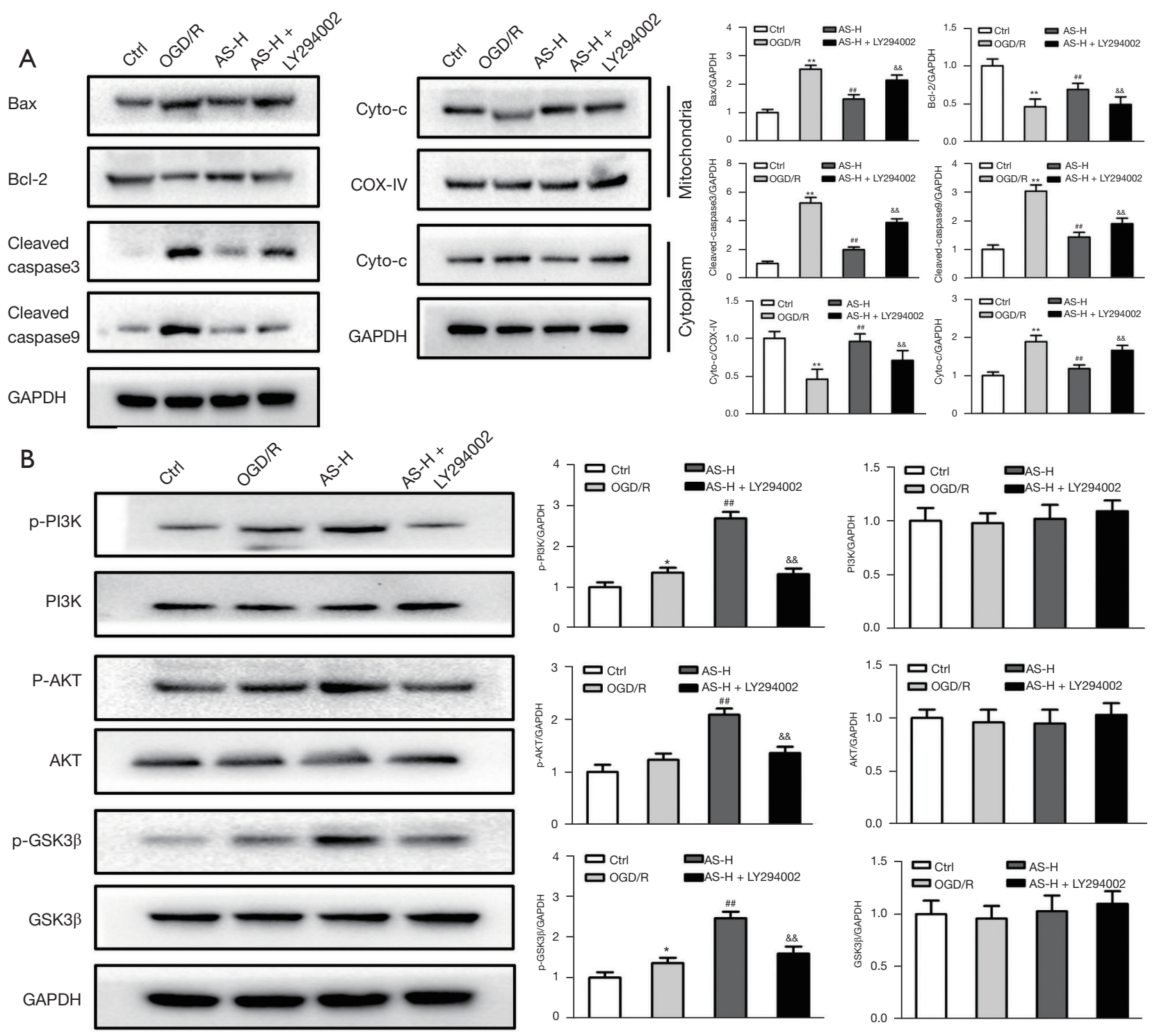

Figure 6 LY294002 partially antagonized the regulation of protein expression by AS. (A) The expression levels of apoptosis-related proteins were measured by western blot in HL-1 cells with various treatments. (B) The protein and phosphorylation levels of PI3K, $A K T$, and GSK3 $\beta$ were detected by western blot in differently treated HL-1 cells. The band intensities were quantified using Image J software and normalized to GAPDH or COX-IV. Error bars indicate means $\pm \mathrm{SD}$ of 3 independent experiments. ${ }^{*} \mathrm{P}<0.05$ or ${ }^{* *} \mathrm{P}<0.01$, OGD/R group $v s$. control group; ${ }^{\# \#} \mathrm{P}<0.01$, AS-H group vs. OGD/R group; ${ }^{\& \&} \mathrm{P}<0.01$, AS-H + LY294002 group vs. AS-H group. AS, asiaticoside; OGD/R, oxygenglucose deprivation/reperfusion; SD, standard deviation; L, low-dose; H, high-dose.

AS on MI/R injury. It was shown that AS protected against $\mathrm{MI} / \mathrm{R}$ and reperfusion injury by attenuating oxidative stress and apoptosis via activating the $P I 3 K-A K T-G S K 3 \beta$ pathway in vivo and vitro. Therefore, AS could be a novel therapeutic drug in further clinical application.

\section{Acknowledgments}

Funding: This work was supported by Science and Technology Research Project of Jiangxi Provincial Department of Education (GJJ201541). 


\section{Footnote}

Reporting Checklist: The authors have completed the ARRIVE reporting checklist. Available at https://atm. amegroups.com/article/view/10.21037/atm-21-6667/rc

Data Sharing Statement: Available at https://atm.amegroups. com/article/view/10.21037/atm-21-6667/dss

Conflicts of Interest: All authors have completed the ICMJE uniform disclosure form (available at https://atm. amegroups.com/article/view/10.21037/atm-21-6667/coif). The authors have no conflicts of interest to declare.

Ethical Statement: The authors are accountable for all aspects of the work in ensuring that questions related to the accuracy or integrity of any part of the work are appropriately investigated and resolved. Experiments were performed under a project license (No. LLSC-2020090801) granted by the Gannan Medical University Institutional Animal Care and Use Committee, in compliance with the Guide for the Humane Treatment of Laboratory Animals (Ministry of Science and Technology of the People's Republic of China, Policy No. 2006398) for the care and use of animals.

Open Access Statement: This is an Open Access article distributed in accordance with the Creative Commons Attribution-NonCommercial-NoDerivs 4.0 International License (CC BY-NC-ND 4.0), which permits the noncommercial replication and distribution of the article with the strict proviso that no changes or edits are made and the original work is properly cited (including links to both the formal publication through the relevant DOI and the license). See: https://creativecommons.org/licenses/by-nc-nd/4.0/.

\section{References}

1. Zhang H, Gong G, Wang P, et al. Heart specific knockout of Ndufs4 ameliorates ischemia reperfusion injury. J Mol Cell Cardiol 2018;123:38-45.

2. Moens AL, Claeys MJ, Timmermans JP, et al. Myocardial ischemia/reperfusion-injury, a clinical view on a complex pathophysiological process. Int J Cardiol 2005;100:179-90.

3. Zhou YH, Han QF, Gao L, et al. HMGB1 Protects the Heart Against Ischemia-Reperfusion Injury via PI3K/AkT Pathway-Mediated Upregulation of VEGF Expression. Front Physiol 2020;10:1595.
4. Ibáñez B, Heusch G, Ovize M, et al. Evolving therapies for myocardial ischemia/reperfusion injury. J Am Coll Cardiol 2015;65:1454-71.

5. Garcia-Dorado D, Rodríguez-Sinovas A, Ruiz-Meana M, et al. Protection against myocardial ischemia-reperfusion injury in clinical practice. Rev Esp Cardiol (Engl Ed) 2014;67:394-404.

6. Singh B, Rastogi RP. A reinvestigation of the triterpenes of Centella asiatica. Phytochemistry 1969;8:917-21.

7. Yousaf S, Hanif MA, Rehman R, et al. Chapter 32 - Indian Pennywort. In: Hanif MA, Nawaz H, Khan MM, et al. editors. Medicinal Plants of South Asia. Amsterdam: Elsevier, 2020:423-37.

8. Sunilkumar, Parameshwaraiah S, Shivakumar HG. Evaluation of topical formulations of aqueous extract of Centella asiatica on open wounds in rats. Indian J Exp Biol 1998;36:569-72.

9. Jing L, Haitao W, Qiong W, et al. Anti inflammatory effect of asiaticoside on human umbilical vein endothelial cells induced by ox-LDL. Cytotechnology 2018;70:855-64.

10. Yingchun L, Huihan W, Rong Z, et al. Antitumor Activity of Asiaticoside Against Multiple Myeloma Drug-Resistant Cancer Cells Is Mediated by Autophagy Induction, Activation of Effector Caspases, and Inhibition of Cell Migration, Invasion, and STAT-3 Signaling Pathway. Med Sci Monit 2019;25:1355-61.

11. Luo Y, Fu C, Wang Z, et al. Asiaticoside attenuates the effects of spinal cord injury through antioxidant and anti inflammatory effects, and inhibition of the p38 MAPK mechanism. Mol Med Rep 2015;12:8294-300.

12. Luo J, Zhang T, Zhu C, et al. Asiaticoside might attenuate bleomycin-induced pulmonary fibrosis by activating cAMP and Rap1 signalling pathway assisted by A2AR. J Cell Mol Med 2020;24:8248-61.

13. Zhang C, Chen S, Zhang Z, et al. Asiaticoside Alleviates Cerebral Ischemia-Reperfusion Injury via NOD2/ Mitogen-Activated Protein Kinase (MAPK)/Nuclear Factor kappa B (NF-кB) Signaling Pathway. Med Sci Monit 2020;26:e920325.

14. Zhang J, Yao M, Jia X, et al. Hexokinase II Upregulation Contributes to Asiaticoside-Induced Protection of H9c2 Cardioblasts During Oxygen-Glucose Deprivation/ Reoxygenation. J Cardiovasc Pharmacol 2020;75:84-90.

15. Papaxoinis G, Kotoula V, Alexopoulou Z, et al. Significance of PIK3CA Mutations in Patients with Early Breast Cancer Treated with Adjuvant Chemotherapy: A Hellenic Cooperative Oncology Group (HeCOG) Study. 
PLoS One 2015;10:e0140293.

16. Ibarrola J, Matilla L, Martínez-Martínez E, et al. Myocardial Injury After Ischemia/Reperfusion Is Attenuated By Pharmacological Galectin-3 Inhibition. Sci Rep 2019;9:9607.

17. Li F, Yao J, Hao Q, et al. miRNA-103 promotes chondrocyte apoptosis by down-regulation of Sphingosine kinase- 1 and ameliorates PI3K/AKT pathway in osteoarthritis. Biosci Rep 2019;39:BSR20191255.

18. Ehrhardt C, Ludwig S. A new player in a deadly game: influenza viruses and the PI3K/Akt signalling pathway. Cell Microbiol 2009;11:863-71.

19. Wang S, Lin S, Zhu M, et al. Acupuncture Reduces Apoptosis of Granulosa Cells in Rats with Premature Ovarian Failure Via Restoring the PI3K/Akt Signaling Pathway. Int J Mol Sci 2019;20:6311.

20. Manning BD, Cantley LC. AKT/PKB signaling: navigating downstream. Cell 2007;129:1261-74.

21. Namba T, Kodama R, Moritomo S, et al. Zidovudine, an anti-viral drug, resensitizes gemcitabine-resistant pancreatic cancer cells to gemcitabine by inhibition of the Akt-GSK3 $\beta-S n a i l$ pathway. Cell Death Dis 2015;6:e1795.

22. Liu G, Zhang BF, Hu Q, et al. Syringic acid mitigates myocardial ischemia reperfusion injury by activating the PI3K/Akt/GSK-3 $\beta$ signaling pathway. Biochem Biophys Res Commun 2020;531:242-9.

23. Park JH, Lee TK, Kim DW, et al. Neuroprotective Effects of Salicin in a Gerbil Model of Transient Forebrain Ischemia by Attenuating Oxidative Stress and Activating PI3K/Akt/GSK3 $\beta$ Pathway. Antioxidants (Basel) 2021;10:629.

24. Ke B, Shen XD, Kamo N, et al. $\beta$-catenin regulates innate and adaptive immunity in mouse liver ischemia-reperfusion injury. Hepatology 2013;57:1203-14.

25. Gao E, Lei YH, Shang X, et al. A novel and efficient model of coronary artery ligation and myocardial infarction in the mouse. Circ Res 2010;107:1445-53.

26. Matsuura K, Canfield K, Feng W, et al. Metabolic Regulation of Apoptosis in Cancer. Int Rev Cell Mol Biol 2016;327:43-87.

27. Geng X, Hong Q, Wang W, et al. Biological MembranePacked Mesenchymal Stem Cells Treat Acute Kidney Disease by Ameliorating Mitochondrial-Related Apoptosis. Sci Rep 2017;7:41136.

28. Razali NNM, Ng CT, Fong LY. Cardiovascular Protective Effects of Centella asiatica and Its Triterpenes: A Review. Planta Med 2019;85:1203-15.

29. Liu X, Liu X, Wang R, et al. Circulating microRNAs indicate cardioprotection by sevoflurane inhalation in patients undergoing off-pump coronary artery bypass surgery. Exp Ther Med 2016;11:2270-6.

30. Ohtori S, Takahashi K, Moriya H, et al. TNF-alpha and TNF-alpha receptor type 1 upregulation in glia and neurons after peripheral nerve injury: studies in murine DRG and spinal cord. Spine (Phila Pa 1976) 2004;29:1082-8.

31. Yue Q, Peng Y, Zhao Y, et al. Dual-targeting for brainspecific drug delivery: synthesis and biological evaluation. Drug Deliv 2018;25:426-34.

32. Morimoto H, Takahashi M, Shiba Y, et al. Bone marrow-derived CXCR4+ cells mobilized by macrophage colony-stimulating factor participate in the reduction of infarct area and improvement of cardiac remodeling after myocardial infarction in mice. Am J Pathol 2007;171:755-66.

33. Chatterjee A, Barnard J, Moravec C, et al. Connective tissue growth factor dependent collagen gene expression induced by MAS agonist AR234960 in human cardiac fibroblasts. PLoS One 2017;12:e0190217.

34. Zhang Y, Zhong JF, Qiu H, et al. Epigenomic Reprogramming of Adult Cardiomyocyte-Derived Cardiac Progenitor Cells. Sci Rep 2015;5:17686.

35. Zhu L, Zhou X, Li S, et al. miR 183 5p attenuates cerebral ischemia injury by negatively regulating PTEN. Mol Med Rep 2020;22:3944-54.

36. Hadi NR, Yusif FG, Yousif M, et al. Both castration and goserelin acetate ameliorate myocardial ischemia reperfusion injury and apoptosis in male rats. ISRN Pharmacol 2014;2014:206951.

37. Li JZ, Xie MQ, Mo D, et al. Picroside II protects myocardium from ischemia/reperfusion-induced injury through inhibition of the inflammatory response. Exp Ther Med 2016;12:3507-14.

38. Yu HT, Zhen J, Pang B, et al. Ginsenoside Rg1 ameliorates oxidative stress and myocardial apoptosis in streptozotocin-induced diabetic rats. J Zhejiang Univ Sci B 2015;16:344-54.

39. Li X, Yuan T, Chen D, et al. Cardioprotective Effects of Puerarin-V on Isoproterenol-Induced Myocardial Infarction Mice Is Associated with Regulation of PPAR-Y/ NF-кB Pathway. Molecules 2018;23:3322.

40. Farooque M, Isaksson J, Olsson Y. Improved recovery after spinal cord injury in neuronal nitric oxide synthasedeficient mice but not in TNF-alpha-deficient mice. J Neurotrauma 2001;18:105-14.

41. Feng L, Wang X, Peng F, et al. Walnut Protein 
Hydrolysates Play a Protective Role on Neurotoxicity Induced by d-Galactose and Aluminum Chloride in Mice. Molecules 2018;23:2308.

42. Park JH, Lee NK, Lim HJ, et al. Therapeutic Cell Protective Role of Histochrome under Oxidative Stress in Human Cardiac Progenitor Cells. Mar Drugs 2019;17:368.

43. Cao CM, Zhang Y, Weisleder N, et al. MG53 constitutes a primary determinant of cardiac ischemic preconditioning. Circulation 2010;121:2565-74.

44. Ban K, Cooper AJ, Samuel S, et al. Phosphatidylinositol 3-kinase gamma is a critical mediator of myocardial ischemic and adenosine-mediated preconditioning. Circ Res 2008;103:643-53.

45. Uchiyama T, Engelman RM, Maulik N, et al. Role of Akt signaling in mitochondrial survival pathway triggered by hypoxic preconditioning. Circulation 2004;109:3042-9.

46. Datta SR, Brunet A, Greenberg ME. Cellular survival: a play in three Akts. Genes Dev 1999;13:2905-27.

47. Juhaszova M, Zorov DB, Yaniv Y, et al. Role of glycogen synthase kinase-3beta in cardioprotection. Circ Res 2009;104:1240-52.

48. Tong H, Imahashi K, Steenbergen C, et al. Phosphorylation of glycogen synthase kinase-3beta during preconditioning through a phosphatidylinositol-3kinase--dependent pathway is cardioprotective. Circ Res 2002;90:377-9.

(English Language Editor: J. Jones)
Cite this article as: Zeng X, Yu J, Liu P, Liu Y, Zeng T, Li B. Asiaticoside alleviates cardiomyocyte apoptosis and oxidative stress in myocardial ischemia/reperfusion injury via activating the PI3K-AKT-GSK3 $\beta$ pathway in vivo and in vitro. Ann Transl Med 2022;10(2):69. doi: 10.21037/atm-21-6667 